\title{
Computational fluid dynamics analysis on recurrence of simple coiling intracranial aneurysms with remnant neck
}

\author{
Xilong $\mathrm{Yu}^{1 \dagger}$, Gang Lu ${ }^{1 \dagger}$, Shengzhang Wang ${ }^{2 *}$, Lei Huang ${ }^{1}$, Liang Ge ${ }^{1}$, Jun Wan ${ }^{3}$, Ruoyu Di ${ }^{1}$, Yeqing Jiang ${ }^{1}$ \\ and Xiaolong Zhang ${ }^{1 *}$
}

\begin{abstract}
Background: Hemodynamic factors are considered to play an important role in the initiation and recurrence of cerebral aneurysms. However, hemodynamic characteristics leading to the recurrence of aneurysms is not well recognized. This study analyzes the hemodynamics of simple-coiling and remnant-neck aneurysms, and discusses the impact of hemodynamic factors on the recurrence of cerebral aneurysms.

Methods: This study selected three simple-coiled and remnant-neck aneurysms with at least once angiographic follow-up. The follow-up was conducted by 3D rotational DSA and the 3D reconstruction of the vessel. The aneurysms located on ophthalmic segment of ICA, including one recurrent case and two stable cases. We used a porous medium model to simulate and analyze the hemodynamics of aneurysms.

Results: For the recanalized aneurysm, in the immediate post-operative period, the WSS at the remnant neck decreased, low blood flow velocity and stagnant regions were found in the aneurysm sac. In the first follow-up, compared to immediately post-operative, at the residual neck, the WSS was higher, the flow impingement was stronger. In the sac, the stagnation area decreased and its contour limit shrank into the aneurysm. However, in the second follow-up, compared to the previous follow-up, at the remnant neck, the WSS was lower, the flow impingement weaker, while in the sac the stagnant region expanded.
\end{abstract}

Conclusions: Aneurysm recurrence could occur in two phases: early coil compaction resulting from blood flow impingement and later vessel remodeling due to hemodynamic effects.

Keywords: Cerebral aneurysm, Remnant neck, Recanalization, Computational fluid dynamics, Embolization Abbreviations: 3D, Three- dimensional; CFD, Computational fluid dynamics; DSA, Digital subtraction angiography; ICA, Intracranial carotid artery; WSS, Wall shear stress

\section{Background}

Endovascular embolization has been widely used as a safe and effective treatment method for intracranial aneurysms. However, the residual neck of intracranial aneurysms can easily relapse after coil embolization [1]. Recurrence is usually influenced by multiple

\footnotetext{
* Correspondence: szwang@fudan.edu.cn; xiaolongzhang@fudan.edu.cn ${ }^{\dagger}$ Equal contributors

2Department of Mechanics and Engineering Science, Fudan University, Shanghai 200433, China

1 Department of Radiology, Huashan Hospital Affiliated to Fudan University, 12 Middle Wulumuqi Road, Shanghai 200040, China

Full list of author information is available at the end of the article
}

factors, and the pathogenesis is still unclear [2-4]. It is a significant clinical problem, waiting to be addressed by the advancement of endovascular techniques. With the rapid development of hydromechanics and numerical simulation of computational fluid dynamics and its close connection with clinical work, more and more studies increasingly suggest that intracranial aneurysm hemodynamic parameters may be related to recurrence of aneurysms after coil embolization [5-13]. As aneurysm remnant may still carry a small risk of rupture [14], it is important to study the underlying mechanisms of aneurysm recurrence after coiling. So it is important 
to study the underlying recurrence mechanism of the remnant-neck aneurysms treated with simple coiling.

Meanwhile, recently most hydrodynamic research only simulates the hemodynamic characteristics of the vessel and the aneurysm remnant-neck after coil embolization $[15,16]$. However, these studies did not consider the percolation in the coils after embolization, and hence the conclusion is different with the actual situation.

Our study used a porous medium model to simulate the hydrodynamics of three aneurysms with a remnantneck after coil embolization. We used the porous medium to simulate the coiled aneurysm, and pure fluid to simulate the parent artery, then calculated and simulated the blood flow with CFD. This study analyzed the hemodynamic changes in the pre-operative, the immediate post-operative period and for the two follow-up periods in order to discuss the hemodynamic mechanism of aneurysm recurrence.

\section{Methods \\ Clinical and image data}

Three cases of remnant-neck aneurysms treated with simple coiling were collected, and all of the DSA images meet the quality requirements for CFD purposes. The cases were followed up by the rotational DSA and the $3 \mathrm{D}$ reconstruction of the vessel. If the remnant neck increased compared with the immediate post-operative period, we defined it as recurrence. One of the cases was found to have recanalization on follow-up, whereas the other two aneurysms were stable. The recurrence case had two follow-up visits, the first visit was 6 months after coil embolization. The DSA showed that the

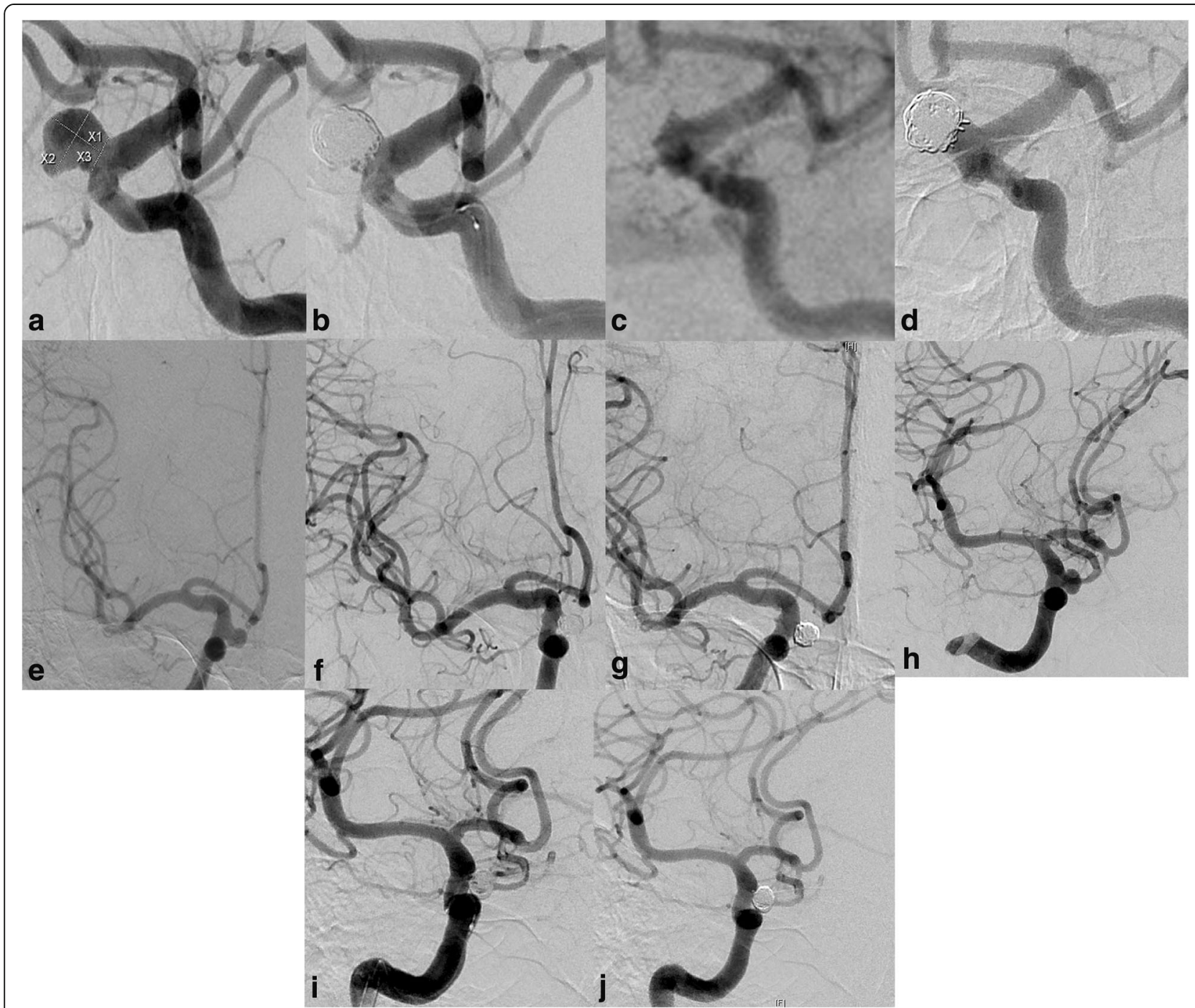

Fig. 1 Digital subtraction angiography (DSA) of aneurysms a Pre-operatively, $\mathbf{b}$ the immediate post-operative period, $\mathbf{c} 6$-month follow-up and $\mathbf{d}$ 18-month follow-up angiography of recanalized case 1; e Pre-operatively, $\mathbf{f}$ the immediate post-operative period and $\mathbf{g}$ 7-month follow-up angiography of stable case $1 ; \mathbf{h}$ Pre-operatively, $\mathbf{i}$ the immediate post-operative period and $\mathbf{j}$ 6-month follow-up of stable case 2 
aneurysm had not recurred. The second visit was 18 months after initial coil embolization, the angiography revealed re-enlargement of the remnant neck as the aneurysm recurred. The other two stable cases had one follow-up visit, which was 6 and 7 months respectively after procedure. The angiography images before and after coil embolization are shown in Fig. 1.

\section{Cerebral aneurysm and coil embolization modeling}

Patient-specific 3D DSA data was obtained from imaging studies performed as diagnostic angiograms. The angiograms were acquired on interventional monoplane C-arm systems (Axiom Artis Zeego, Siemens Medical Solutions, Erlangen, Germany). All the images acquired were then transferred to the angiography workstation (Siemens Syngo X-workplace). Using Siemens Leonardo image post-processing with Inspace software, dual 3D reconstructions of aneurysm and cerebral artery were created. After selecting a volume of interest containing aneurysm and adjacent vessels, the workstation exported the reconstructed 3D image into an STL (stereolithographic) file. The STL file was then imported into Geomagic Studio 11.0 software to repair, cut and smooth. Using the method proposed by Kakalis et al. [17], the whole vessel volume was divided into porous medium and pure fluid regions. We modeled the system of the detachable coils in the aneurysm volume as a porous medium, while the parent artery and remnant neck were modeled as the pure fluid part, For the porous region, the volume porosity $\phi$ of the porous region represents the volume occupied by pore to the total volume of the aneurysm sac and is calculated according to the formula

$$
\phi=1-\frac{\sum_{i=1}^{N} V c, i}{V \text { an }}
$$

Where $\mathrm{N}$ is the number of coils released into the aneurysm, $V \mathrm{c}, i$ is the volume of $i$ th coil, and Van is the total volume of the aneurysm.

In Fig. 2, we illustrated the partition of the aneurysm volume into porous and pure fluid regions. The red region is filled with the porous medium and corresponds to the coiled part of the aneurysm whereas the blue one to the pure fluid part.

\section{Hemodynamic modeling}

The STL (stereolithography) format file was then imported into ANSYS ICEM CFD_13.0 software to create volume grids. For the coiled aneurysm model, the geometry of the original aneurysm was divided into two parts, the aneurysm sac and the parent artery by the interface at the aneurysm neck, but the parameters for the mesh generation of the three parts were the same. The tetrahedral meshes were created with a maximum

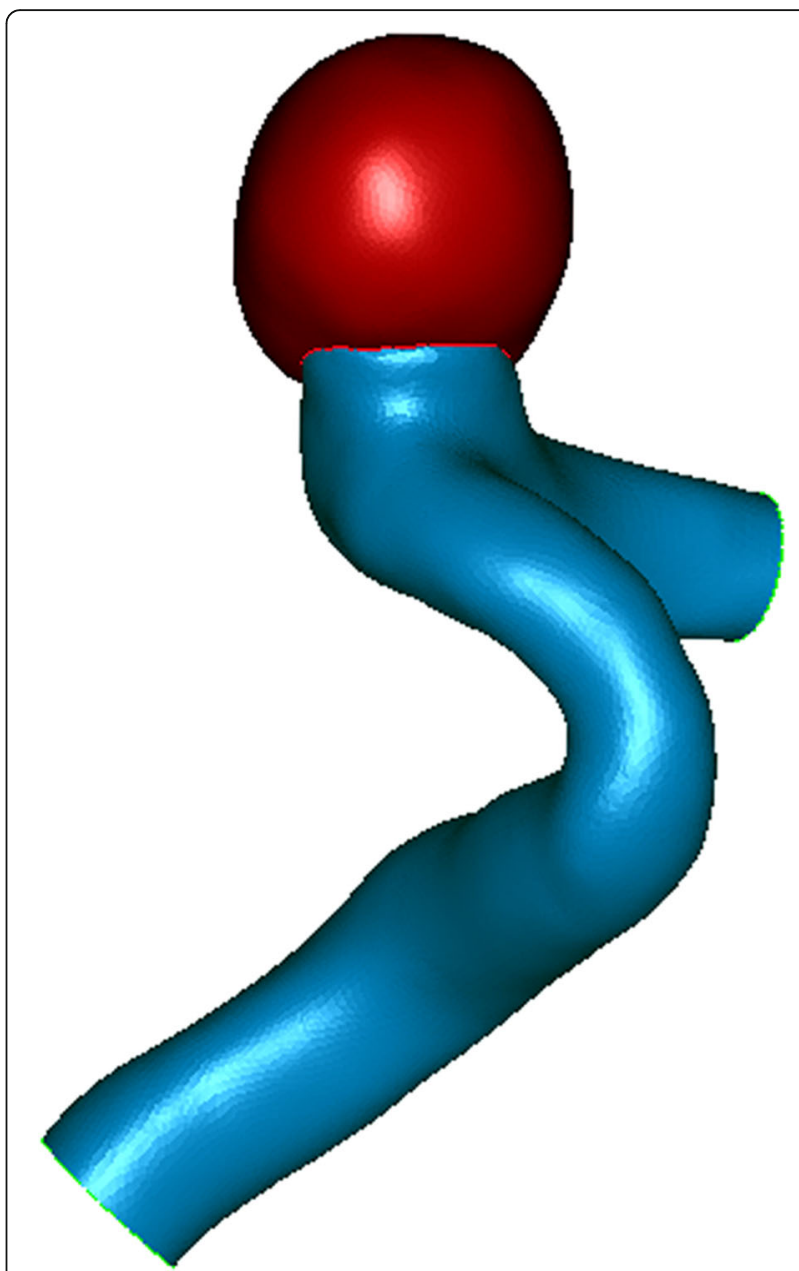

Fig. 2 Partition of the aneurysm volume into a porous medium (red) and a pure fluid part (blue)

mesh size of $0.2 \mathrm{~mm}$. The meshes were imported into the commercial CFD package ANSYS CFX_13.0 (Ansys Inc., Canonsburg, PA, USA) to simulate the blood flow in the untreated aneurysm model and treated aneurysm models. The treated aneurysm was divided into a porous medium region and pure flow region, using a porous medium to stimulate the coiled aneurysm sac, and using the pure fluid to simulate the parent artery and the remnant neck. As is illustrated in Fig. 3, the red area was filled with the porous medium and corresponds to the coiled part of the aneurysm, while the blue region corresponded to the blood flow in the remnant neck, while the green corresponded to the blood flow in the parent artery. Blood flow was considered an incompressible and laminar fluid and satisfied the Navier-Stokes equation and continuity equation. The density and dynamic viscosity of blood were specified as $1060 \mathrm{~kg} / \mathrm{m}^{3}$ and $0.004 \mathrm{~N} / \mathrm{m}^{2}$. The vessel wall was assumed rigid and no-slip, while the pulsatile velocity boundary condition was imposed at the model inlet by using a superposition 


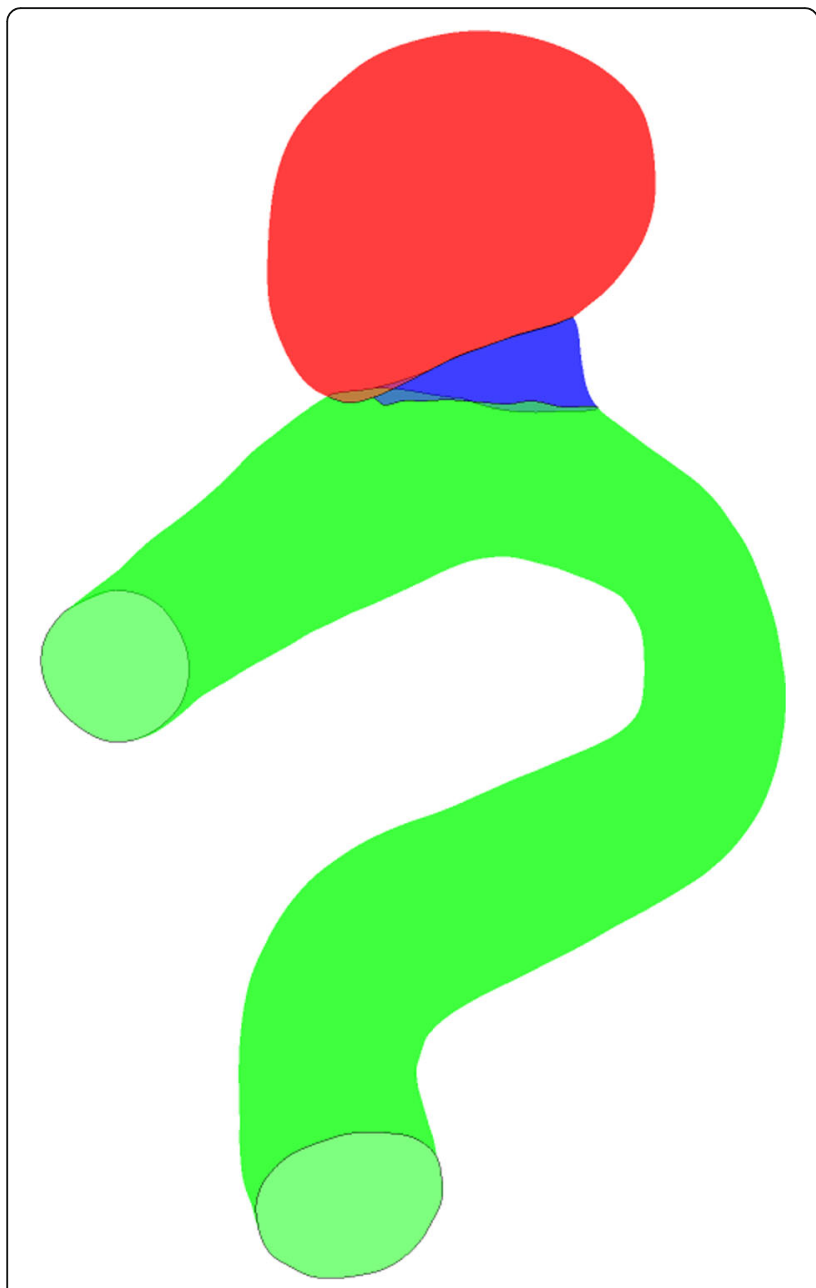

Fig. 3 The simulation model of the aneurysms

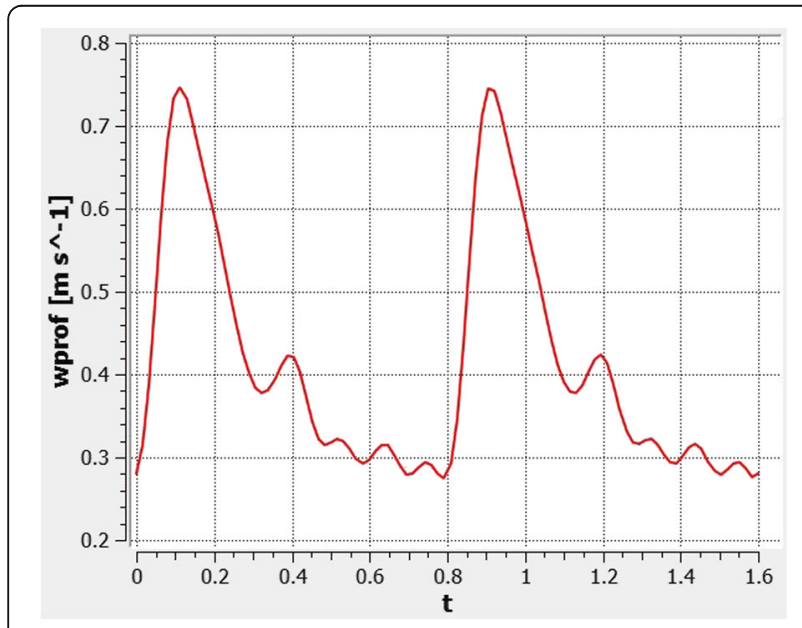

Fig. 4 The velocity waveform image of the model inlet of Womersley velocity profiles. The waveform picture is illustrated in Fig. 4. The pressure boundary condition was imposed at the model outlet, and the initial Pressure was $8000 \mathrm{~Pa}$. Two cardiac cycles of the blood flow were performed for each simulation, with the data in the second cycle used as the final result. The blood flow in the convergence criteria in the simulation was that the residual of velocity and mass were less than $1 \mathrm{e}-5$.

\section{Results}

Numerical simulation of blood flow was conducted under corresponding state. The streamlines, the distributions of the wall shear stress (WSS) and the velocity contours of aneurysms were acquired.

\section{Streamlines}

Figure 5 respectively shows the streamlines of the coiled aneurysm at the systolic peak of the secondary cardiac cycle before and after coil embolization

Pre-operatively, part of the blood flow entered the aneurysm sac from the distal side of the neck and formed a large and complex vortex inside aneurysm. In the Immediate post-operative period, there was still some minor blood flow in the aneurysm, which entered the aneurysm from the distal side of the remnant neck, but the blood flow in the aneurysm was less than the untreated aneurysm. In the two follow-ups, compared to the immediate post-operative period, the blood flow in the aneurysm had no obvious change, there was still some slight blood flow in the aneurysm. But for recanalized case 1 , high velocity and dense streamlines could be demonstrated by the enlargement of the remnant neck. Luo B et al. [16] assumed, after partial occlusion, that the blood inflow did not enter the aneurysm sac. They used a model of the parent artery for hemodynamic analysis after coil embolization, which was equivalent to directly deleting the aneurysm and did not correspond to the actual blood flow situation. In this study, we used a porous media model to simulate the blood flow in the aneurysm, and more accurately simulated the blood flow.

\section{Distributions of the wall shear stress}

Figure 6 shows the distributions of the wall shear stress at the systolic peak of the cardiac cycle before and after coil embolization respectively

Pre-operatively, the local WSS on the remnant neck was greater than in other regions of the aneurysm. In the immediate post-operative period, the WSS on the remnant neck of the aneurysm decreased, but it was still higher than in other regions of the aneurysm. In the first follow-up, for recanalized case, compared to the immediate post-operative period, the local WSS at the remnant neck had increased; for stable cases, the local WSS at the remnant neck was same as the previous 


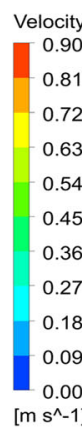

$r$

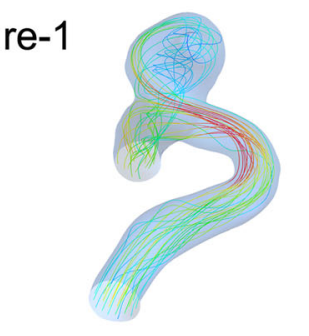

a

st-1

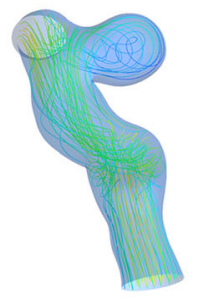

e

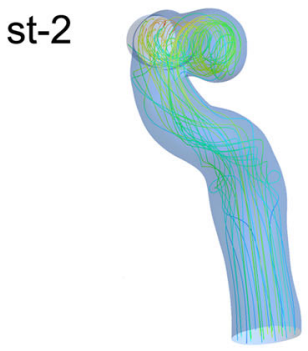

h

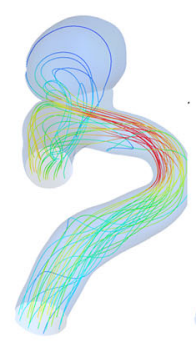

b

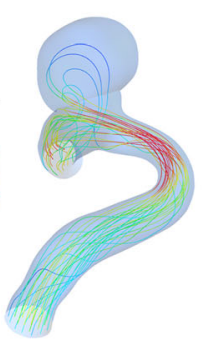

C

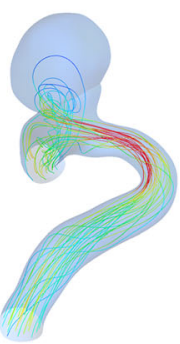

d

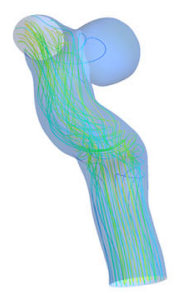

g

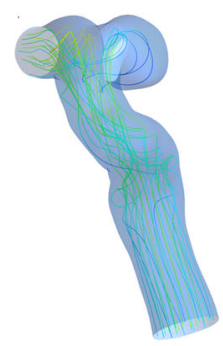

i

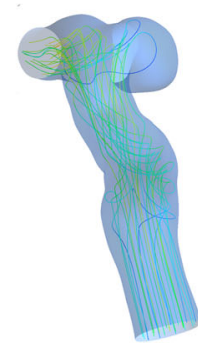

j

Fig. 5 Streamlines of the aneurysm at the systolic peak ( $t=0.2 \mathrm{~s}$ ). Pre-operatively, the blood flow entered the aneurysm sac and formed a large and complex vortex inside aneurysm. In the immediate post-operative period, some minor blood flow entered into the aneurysm). In the two follow-ups, some slight blood flow still entered into in the aneurysm, while high velocity and dense streamlines could be observed at the remnant neck of the recanalized case. For the panel inside the image of Fig. 5 , the red region on the top responds to high velocity, while the blue region at the bottom responds to low velocity

(Table 1). In the second follow-up, for the recanalized case the local WSS at remnant neck of the aneurysm was lower than the previous follow-up, and still higher than in other regions of the aneurysm.

\section{The velocity contours on the longitudinal section}

Figure 7 shows the velocity contours on the longitudinal section for the coiled aneurysm at $0.2 \mathrm{~s}$ of the cardiac cycle (at the systolic peak )in the pre-operative, immediate post-operative, and two follow-up periods respectively

Pre-operatively, the blood flow velocity was rapid in the sac, and no stagnation region was formed in the recanalized and stable aneurysms. In the immediate post-operative period, the velocity became lower in most of the aneurysm sac, and then formed a stagnant region.
While for the recanalized case, an obvious flow impingement region could be observed in a wide area at the remnant neck. In the first follow-up, for the recanalized case, the flow impingement was stronger than the immediate post-operative period, the impingement region was wide, while the stagnation area in the aneurysm was reduced; for stable cases, the velocity had no obvious change (Table 1). In the second follow-up, the flow impingement in the recanalized case became weaker than in the first follow-up.

\section{Discussion}

Recently, hemodynamic research mainly simulated the blood flow characteristics of the remnant neck without considering the coils [16]. However, even when an 

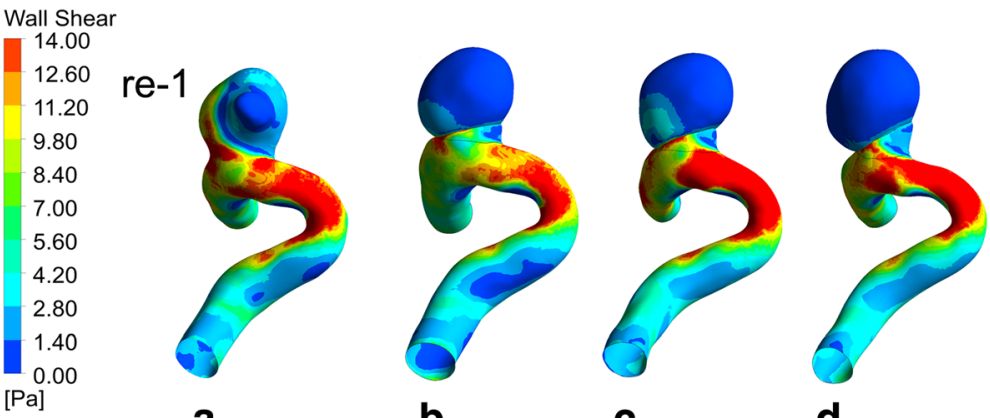

a

b

C

d
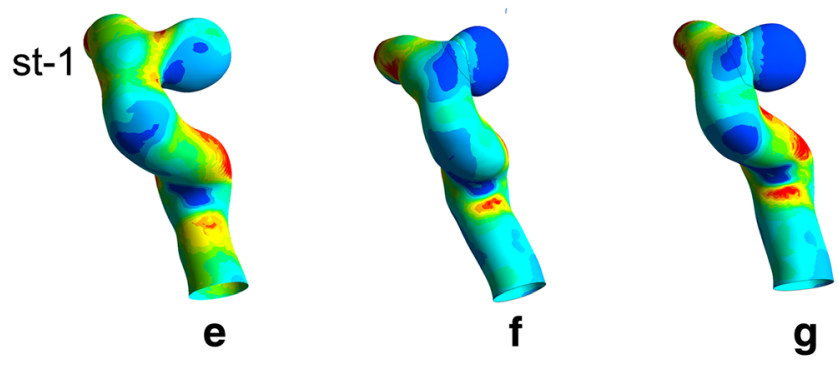

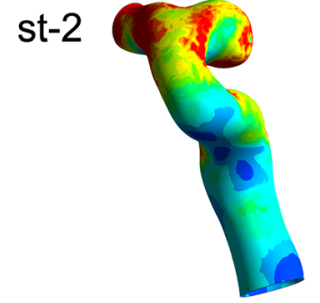

h

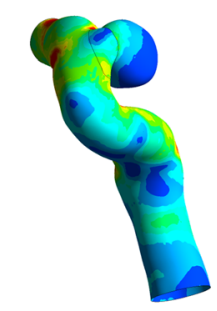

i

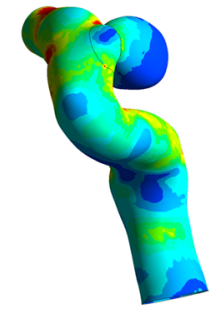

j

Fig. 6 The Wall Shear Stress Contours distributions of the aneurysm at the systolic peak $(t=0.2 \mathrm{~s}$ ). Pre-operatively, the local WSS on the remnant neck was greater than that in other regions of the aneurysm. In the immediate post-operative period, the local WSS on the remnant neck decreased. In the first follow-up, compared to the immediate post-operative period, high local WSS at the remnant neck of recanalized case was observed. In the second follow-up, high local WSS at remnant neck of the recanalized case was also can be found. For the panel inside the image of Fig. 6, the red region on the top responds to high wall shear stress, while the blue region at the bottom responds to low wall shear stress

aneurysm was completely embolized, the packing density might be only $25-35 \%$ [18]. Though the aneurysm displayed no contrast material, in the immediate postoperative period, blood flow percolation could still occur in the aneurysm pouch. Therefore their method did not

Table 1 Magnitude of Wall Shear Stress $(\mathrm{Pa})$ and velocity $(\mathrm{m} / \mathrm{s})$

\begin{tabular}{lllllllll}
\hline & \multicolumn{3}{l}{ Max WSS } & & & Max velocity & \\
\cline { 2 - 4 } & Re-1 & St-1 & St-2 & & Re-1 & St-1 & St-2 \\
\hline Pre-operative & 23.364 & 14.707 & 24.581 & & 0.591 & 0.444 & 0.313 \\
Post-operative & 16.631 & 7.466 & 8.053 & & 0.429 & 0.217 & 0.114 \\
First follow-up & 21.784 & 7.734 & 9.275 & & 0.469 & 0.219 & 0.152 \\
Second follow-up & 18.740 & & & & 0.343 & & \\
\hline
\end{tabular}

WSS indicates wall shear stress, Re-1 recurred case 1, St-1 stable case 1, St-2 stable case 2 accord with the actual situation. Our study used a porous medium model as the equivalent of the coils [19], and combined with the reconstruction of the parent artery to simulate the hemodynamic characteristics of the aneurysm obtaining a more accurate result. Meanwhile, the porous model required the uniformity of the simulation material. In this study, in the immediate post-operative period, the velocity of the blood flow surrounding the coils was high, so that no thrombosis was formed in the aneurysm. As the material in the aneurysm approximated homogeneous distribution, it was reasonable to model the aneurysm with the porous medium. In the two follow-ups, significant thrombosis formed in the aneurysm, resulting in a change of porosity, so that the porous medium may not very accurately model the blood flow, while there 

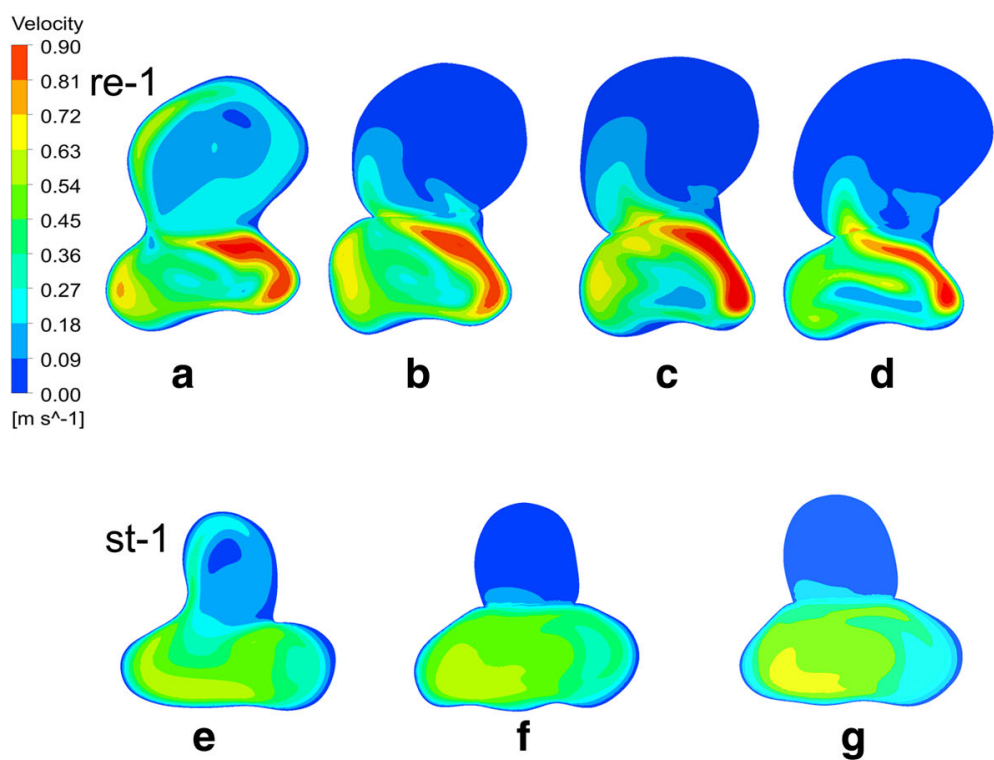

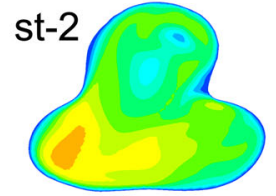

$\mathbf{h}$

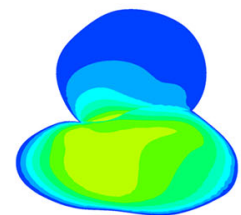

i

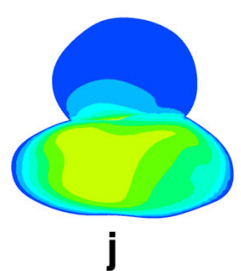

Fig. 7 The Velocity Contours on the Longitudinal Section at the systolic peak $(t=0.2 \mathrm{~s})$. Pre-operatively, the blood flow velocity was rapid in the aneurysm sac without stagnation region. In the immediate post-operative period, the velocity in the sac become lower, and formed a stagnation region $(V<0.1 \mathrm{~m} / \mathrm{s})$. An obvious flow impingement region was observed in the remnant neck of the recanalized aneurysm. In the first follow-up, the flow impingement at the remnant neck of recanalized aneurysm became stronger and formed a wide area, while the stagnation area had reduced. In the second follow-up, the flow impingement became weaker than the previous follow-up and the impingement region became narrow, while the stagnation region enlarged. For the panel inside the image of Fig. 7 , the red region on the top responds to high velocity, while the blue region at the bottom responds to low velocity

was also percolation in the aneurysm. So the porous medium simulation largely replicated the blood flow situation, and the hemodynamic results were relatively accurate.

Sluzewski M [14] et al. demonstrated that coil compaction, resulting from high blood flow impingement, was an important factor in aneurysm recurrence. Some studies had also found that hemodynamic factors, such as high WSS and velocity, were highly related to aneurysm recurrence $[16,20]$. In this study, for recanalized case, in the immediate post-operative period, the form of the aneurysm had no obvious change. However, a sizeable blood stagnation region was formed in the sac, and the WSS on the remnant neck decreased. In the first followup, the remnant neck area had no obvious change, the WSS on the remnant neck was higher than the immediate post-operative period, the flow impingement was stronger, while the impingement region was wide, and the blood stagnation region decreased. In the second follow-up, the remnant neck of the aneurysm enlarged, the aneurysm recurred, and while the WSS was lower on the remnant neck and aneurysm sac than the previous follow-up, the blood stagnation region became larger. For the recanalized case the WSS and velocity underwent dynamic changes during the aneurysm recurrence process after embolization, which influenced the progress of the aneurysm outcome. In conclusion this aneurysm recurrence process can be divided into two phases: in the first phase (from the immediate post-operative period to the first follow-up) the aneurysm did not recur, the coils experienced only minor compaction, resulting in local high blood flow velocity and a decrease in the blood stagnation area, the WSS on the remnant neck increased. In the second phase (from the first follow-up to the second followup) high WSS on the remnant neck resulted in the regrowth of the remnant neck and aneurysm recurrence.

In our study, for recurrent case 1, it was stable in the 6-month follow-up, but had recanalized in the 18-month 
follow up. For recurrent case 2, it recurred in the first follow-up on seventh month after procedure. Therefore, the different interval in different patients between the first follow-up and the second follow-up may induce much influence on the results. And it is necessary to conduct long-term follow-up for remnant-neck aneurysms. In clinical practice, the hemodynamic features of the intracranial aneurysms can also provide some references for the treatment and follow-up of the intracranial aneurysms.

The porous medium model also has some limitations, in the immediate post-operative period, the velocity of the blood flow surrounding the coils was high, so that thrombosis was difficult to be formed in the aneurysm. It is relatively reasonable to model the aneurysm with the porous medium. However, in the two follow-ups, significant thrombosis formed in the aneurysm, resulting in a gradual decrease of porosity, we could not accurately describe the thrombosis and calculate the porosity, but we gave a rough estimate of the porosity of the followup. Simultaneously this model represented blood flow in the aneurysm sac after coil embolization, it might be more reasonable than the previous method which directly deleted the aneurysm.

\section{Conclusion}

This aneurysm recurrence did not result solely from pure coil compaction or vessel reconstruction caused by hemodynamic change, but was rather the result of these two factors working in concert in two stages. In the first phase, high velocity blood flow impingement on the coils resulted in coil compaction, and the aneurysm did not noticeably recur, while hemodynamic parameters significantly changed. In the second phase, the coils were stable, while high WSS for a long period at the remnant neck resulted in vessel reconstruction leading to aneurysm recurrence.

\section{Acknowledgment}

We gratefully acknowledge John Bominghan for English review.

\section{Funding}

This work is supported by the National Science Foundation of China (grant81371308) and the health and family planning in shanghai city committee projects (grant 20134088).

\section{Availability of data and material}

All relevant data of this manuscript can be freely available. And all of the data is linked in https://drive.google.com/ open?id=0BwCpEKrOvwY1NU9scm8xdkIPRGc.

\section{Authors' contributions}

$X L Y$ carried out aneurysmal model reconstruction and CFD calculation, and drafted the manuscript; GL carried out aneurysmal model reconstruction and CFD calculation; SZ W carried out critical revision of the manuscript for important intellectual content.; LH analyzed and interpreted the data; $\mathrm{LG}$ analyzed and interpreted the data; JW acquired the data; RY D carried out aneurysmal model reconstruction; YQ J carried out aneurysmal model reconstruction; XL Z conceived and designed the research, analyzed and interpreted the data, and handled funding and supervision. All authors read and approved the final manuscript.

\section{Author's information}

XL Y: M.D, Department of Radiology, Huashan Hospital Affiliated to Fudan University, Shanghai 200040, China.

GL: PH.D, Department of Radiology, Huashan Hospital Affiliated to Fudan University, Shanghai 200040, China.

SZ W: M.D, Department of Mechanics and Engineering Science, Fudan University, Shanghai 200433, China

LH: M.D, Department of Radiology, Huashan Hospital Affiliated to Fudan University, Shanghai 200040, China

LG: M.D, Department of Radiology, Huashan Hospital Affiliated to Fudan University, Shanghai 200040, China.

JW: M.D, Department of radiology, Jing'an District Centre Hospital, Shanghai 200040, China.

RY D, M.D, Department of Radiology, Huashan Hospital Affiliated to Fudan University, Shanghai 200040, China.

YQ J, M.D, Department of Radiology, Huashan Hospital Affiliated to Fudan University, Shanghai 200040, China.

XL Z, PH.D, Department of Radiology, Huashan Hospital Affiliated to Fudan University, Shanghai 200040, China.

\section{Competing interests}

The authors declare that they have no competing interests.

\section{Consent for publication}

All of the individual person's data in the manuscript has been consented to publish which was obtained from the person in this study.

\section{Ethics approval and consent to participate}

This study on ethics has been approved and consented by the Medical Ethics Committee of Huashan Hospital.

\section{Sponsored by}

National Nature Science Foundation of China (Grant No. 81371308)

\section{Author details}

'Department of Radiology, Huashan Hospital Affiliated to Fudan University, 12 Middle Wulumuqi Road, Shanghai 200040, China. ${ }^{2}$ Department of Mechanics and Engineering Science, Fudan University, Shanghai 200433, China. ${ }^{3}$ Department of radiology, Jing'an District Centre Hospital, Shanghai 200040, China.

Received: 21 February 2016 Accepted: 26 August 2016

Published online: 18 October 2016

\section{References}

1. Nohra C, Cory D. Long-term catheter angiography after aneurysm coil therapy: results of 209 patients and predictors of delayed recurrence and retreatment. J Neurosurg. 2014;121:1102-6.

2. Kai Y, Hamada J, Kuratsu J, et al. Evaluation of the stability of small ruptured aneurysms with a small neck after embolization with Guglielmi detachable coils: Correlation between coil packing ratio and coil compaction. Neurosurgery. 2005;56:785-92.

3. Jin S-C, Kwon O-K, Park H, et al. Simple coiling using single or multiple catheters without balloons or stents in middle cerebral artery bifurcation aneurysms. Neuroradiology. 2013;55:321-6.

4. Gallas S, Pasco A, Cottier JP, et al. A multicenter study of 705 ruptured intracranial aneurysms treated with guglielmi detachable coils. AJNR Am J Neuroradiol. 2005;26:1723-31.

5. Shengzhang Wang*, Jialiang Chen, Xiaolong Zhang, et al. Non-newtonian computational hemodynamics in two patient-specific cerebral aneurysms with daughter saccules. J Hydrodynamics. 2010;22:639-646.

6. Lu G, Zhang XL, Geng DY, et al. Influence of hemodynamic factor on rupture of intracranial aneurysm: patient-specific $3 \mathrm{~d}$ mirror aneurysms model computational fluid dynamics simulation. AJNR Am J Neuroradiol. 2011;32:1255-61.

7. Chatziprodromou I, Tricoli A, Poulikakos D, Ventikos Y. Haemodynamics and wall remodelling of a growing cerebral aneurysm: a computational model. J Biomech. 2007:40:412-26.

8. Low M, Perktold K, Raunig R. Hemodynamics in rigid and distensible saccular aneurysms: a numerical study of pulsatile flow characteristics. Biorheology. 1993;30:287-98. 
9. Jou LD, Wong G, Dispensa $B$, et al. Correlation between luminal geometry changes and hemodynamics in fusiform intracranial aneurysms. AJNR Am J Neuroradiol. 2005;26:2357-63.

10. Acevedo-Bolton G, Jou LD, Dispensa BP, et al. Estimating the hemodynamic impact of interventional treatments of aneurysms: numerical simulation with experimental validation: technical case report. Neurosurgery. 2006;59:E429-30.

11. Sugiyama SI, Meng H, Funamoto K, et al. Hemodynamic analysis of growing intracranial aneurysms arising from a posterior inferior cerebellar artery. World Neurosurg. 2012;78:462-68.

12. Shojima M, Oshima M, Takagi $\mathrm{K}$, et al. Magnitude and role of wall shear stress on cerebral aneurysm: computational fluid dynamic study of 20 middle cerebral artery aneurysms. Stroke. 2004;35:2500-5.

13. Ford MD, Nikolov HN, Milner JS, et al. Piv-measured versus CFD-predicted flow dynamics in anatomically realistic cerebral aneurysm models. J Biomech Eng. 2008;130:210-15.

14. Sluzewski M, Rooij W, Slob M, et al. Relation between aneurysm volume, packing and compaction in 145 cerebral aneurysms treated with coils. Radiology. 2004;231:653-8

15. Hasan DM, Nadareyshvili Al, Hoppe AL, et al. Cerebral aneurysm sac growth as the etiology of recurrence after successful coil embolization. Stroke. 2012;43:86-868.

16. Luo $B$, Yang $X$, Wang $S$, et al. High shear stress and flow velocity in partially occluded aneurysms prone to recanalization. Stroke. 2011;42:745-53.

17. Kakalis NMP, Mitsos AP, Byrne JV, et al. The haemodynamics of endovascular aneurysm treatment: A computational modeling approach for estimating the influence of multiple coil deployment. IEEE Transmed Imaging. 2008;27:814-24.

18 Choi DS, Kim MC, Terbrugge KG, et al. Clinical and angiographic long-term follow-up of completely coiled intracranial aneurysms using endovascular technique. J Neurosurg. 2010;112:575-81.

19 Mitsos AP, Kakalis NMP, Ventikos YP, et al. Haemodynamic simulation of aneurysm coiling in an anatomically accurate computational fluid dynamics model: Technical note. Neuroradiology. 2008;50:341-7.

20 Keiko I, Hitomi A, Naomi H, et al. Computational fluid dynamic analysis following recurrence of cerebral aneurysm after coil embolization. AJNS. 2012; :109-15

\section{Submit your next manuscript to BioMed Central and we will help you at every step:}

- We accept pre-submission inquiries

- Our selector tool helps you to find the most relevant journal

- We provide round the clock customer support

- Convenient online submission

- Thorough peer review

- Inclusion in PubMed and all major indexing services

- Maximum visibility for your research

Submit your manuscript at www.biomedcentral.com/submit

) Biomed Central 